\title{
ON SUPERNILPOTENT RADICALS WITH THE AMITSUR PROPERTY
}

\author{
HALINA FRANCE-JACKSON
}

(Received 11 December 2008)

\begin{abstract}
A radical $\alpha$ has the Amitsur property if $\alpha(A[x])=(\alpha(A[x]) \cap A)[x]$ for all rings $A$. For rings $R \subseteq S$ with the same unity, we call $S$ a finite centralizing extension of $R$ if there exist $b_{1}, b_{2}, \ldots, b_{t} \in S$ such that $S=b_{1} R+b_{2} R+\cdots+b_{t} R$ and $b_{i} r=r b_{i}$ for all $r \in R$ and $i=1,2, \ldots, t$. A radical $\alpha$ is FCEfriendly if $\alpha(S) \cap R \subseteq \alpha(R)$ for any finite centralizing extension $S$ of a ring $R$. We show that if $\alpha$ is a supernilpotent radical whose semisimple class contains the ring $\mathbb{Z}$ of all integers and $\alpha$ is FCE-friendly, then $\alpha$ has the Amitsur property. In this way the Amitsur property of many well-known radicals such as the prime radical, the Jacobson radical, the Brown-McCoy radical, the antisimple radical and the Behrens radical can be established. Moreover, applying this condition, we will show that the upper radical $\mathcal{U}\left(*_{k}\right)$ generated by the essential cover $*_{k}$ of the class $*$ of all $*$-rings has the Amitsur property and $\mathcal{U}\left(*_{k}\right)(A[x])=\mathcal{U}\left(*_{k}\right)(A)[x]$, where a semiprime ring $R$ is called a $*$-ring if the factor ring $R / I$ is prime radical for every nonzero ideal $I$ of $R$. The importance of $*$-rings stems from the fact that a $*$-ring $A$ is Jacobson semisimple if and only if $A$ is a primitive ring.
\end{abstract}

2000 Mathematics subject classification: primary 16N80.

Keywords and phrases: Amitsur property of radicals, polynomial rings, finite centralizing extension, prime radical, essential cover, special class, special and supernilpotent radicals, *-rings, prime heart, pseudo radical.

\section{Introduction}

In this paper all rings are associative and all classes of rings are closed under isomorphisms and contain the one-element ring 0 . The fundamental definitions and properties of radicals can be found in [2] and [7]. A class $\mu$ of rings is called hereditary if $\mu$ is closed under ideals. If $\mu$ is a hereditary class of rings, $\mathcal{U}(\mu)$ denotes the upper radical generated by $\mu$, that is, the class of all rings which have no nonzero homomorphic images in $\mu$. For any class $\mu$ of rings an ideal $I$ of a ring $A$ is called an $\mu$-ideal if the factor ring $A / I$ is in $\mu$. As usual, for a radical $\alpha$, the $\alpha$ radical of a ring $A$ is denoted by $\alpha(A)$ and the class of all $\alpha$-semisimple rings is denoted by $\mathcal{S}(\alpha)$. $\pi$ denotes the class of all prime rings and $\beta=\mathcal{U}(\pi)$ denotes the prime radical.

(C) 2009 Australian Mathematical Publishing Association Inc. 0004-9727/2009 \$16.00 
The notation $I \triangleleft A$ means that $I$ is a two-sided ideal of a ring $A$. Let

$$
\operatorname{Spec}(A)=\{B \triangleleft A \mid A / B \in \pi\}
$$

An ideal $I$ of a ring $A$ is called essential in $A$ if $I \cap J \neq 0$ for any nonzero twosided ideal $J$ of $A$. A ring $A$ is called an essential extension of a ring $I$ if $I$ is an essential ideal of $A$. A class $\mu$ of rings is called essentially closed if $\mu=\mu_{k}$, where $\mu_{k}=\{A \mid A$ is an essential extension of some $I \in \mu\}$ is the essential cover of $\mu$. A hereditary and essentially closed class of prime rings is called a special class and the upper radical generated by a special class is called a special radical. A hereditary radical containing the prime radical $\beta$ is called a supernilpotent radical. Given a ring $A$, the polynomial ring over $A$ in a commuting indeterminate $x$ is denoted by $A[x]$.

An important issue in Radical Theory is to describe the radical of a polynomial ring $A[x]$. We say that a radical $\alpha$ has the Amitsur property if $\alpha(A[x])=(\alpha(A[x]) \cap A)[x]$ for every ring $A$. Although many classical radicals have this important property, in several cases, it is not so easy to determine whether a given radical enjoys the Amitsur property. So it is desirable to have useful criteria for testing the Amistur property of radicals. In this paper we give such a criterion for supernilpotent radicals whose semisimple class contains the ring $\boldsymbol{Z}$ of all integers. In this way the Amitsur property of many important special radicals such as the prime radical, the Jacobson radical, the Brown-McCoy radical, the antisimple radical radical and the Behrens radical can be established.

A semiprime ring $R$ is called a $*$-ring [5] if $R / I \in \beta$ for any nonzero ideal $I$ of $R$. The class of all $*$-rings is denoted by $*$. For a nonzero ring $R$, the intersection $P H_{R}$ (respectively, $S H_{R}$ ) of all the nonzero prime (respectively, semiprime) ideals of $R$ is called the prime (respectively, semiprime) heart of $R$ [5]. In [3] the prime heart of a ring $R$ was called the pseudo radical of $R$ and it was denoted by $\operatorname{ps}(R)$. It was shown in [5] that both, the nonzero semiprime heart of a semiprime ring and the nonzero prime heart of a prime ring are $*$-rings. We also have the following theorem.

THEOREM 1 [5, Theorem 8]. The following conditions are equivalent for a nonzero ring $R$.

(1) $R \in *_{k}$.

(2) $R$ is a semiprime ring with nonzero semiprime heart.

(3) $R$ is a prime ring with nonzero prime heart.

The importance of the class $*_{k}$ is underlined by the two facts that follow.

THEOREM $2[4,8]$. If $R$ is a nonzero *-ring, then the smallest special (respectively, supernilpotent) radical $\hat{l}_{R}$ (respectively, $\bar{l}_{R}$ ) containing $R$ is an atom in the lattice of all special (respectively, supernilpotent) radicals.

Theorem 3 [5, Proposition 2]. If $R \in *_{k}$ and $\mu$ is a special class of rings, then $R \in \mathcal{S}(\mathcal{U}(\mu))$ if and only if $R \in \mu$. Thus, in particular, a ring $R \in *_{k}$ is Jacobson semisimple if and only if $R$ is primitive. 
A long standing open question [6, Problem 1] asks whether $\beta=\mathcal{U}\left(*_{k}\right)$. In this context it is natural to ask whether $\mathcal{U}\left(*_{k}\right)$ behaves similarly to $\beta$ when one takes polynomials. Using our criterion, we will answer this question in the positive by showing that $\mathcal{U}\left(*_{k}\right)$ has the Amitsur property and $\mathcal{U}\left(*_{k}\right)(A[x])=\mathcal{U}\left(*_{k}\right)(A)[x]$.

\section{Main results}

For rings $R \subseteq S$ with the same unity, we say that $S$ is a finite centralizing extension of $R$ generated by $b_{1}, b_{2}, \ldots, b_{t}$ if there exist $b_{1}, b_{2}, \ldots, b_{t} \in S$ such that $S=$ $b_{1} R+b_{2} R+\cdots+b_{t} R$ and $b_{i} r=r b_{i}$ for all $r \in R$ and $i=1,2, \ldots, t$. For example, the field $\mathbb{C}$ of all complex numbers is a centralizing extension of the field $\mathbb{R}$ of all real numbers generated by 1 and $i$.

It was proved in [11, Proposition 3.1] that if $S$ is a finite centralizing extension of $R$ and $\gamma$ denotes the Behrens radical, that is, the upper radical generated by the class of all subdirectly irreducible rings each having a nonzero idempotent in its heart, then $\gamma(S) \cap R \subseteq \gamma(R)$. Moreover, it follows from [9, Corollary 10.2.10 and Corollary 10.4.15] that $\beta(S) \cap R \subseteq \beta(R)$ and $\mathcal{J}(S) \cap R \subseteq \mathcal{J}(R)$, where $\mathcal{J}$ denotes the Jacobson radical and $S$ is a finite centralizing extension of $R$. Inspired by these results, we call a radical $\alpha$ FCE-friendly if $\alpha(S) \cap R \subseteq \alpha(R)$ for any finite centralizing extension $S$ of $R$. Thus $\beta$ and $\mathcal{J}$ are both FCE-friendly.

A radical $\alpha$ is strongly hereditary, if $A \in \alpha$ implies $L \in \alpha$ for every subring $L$ of $A$.

It is a consequence of our next assertion (which is true for all rings, not necessarily with an identity element) that all strongly hereditary radicals are FCE-friendly. In particular, the prime radical, the locally nilpotent radical and the nil radical are all FCE-friendly. See also [1, Lemma 4 and the final paragraph].

LEMMA 4. Let $\alpha$ be a strongly hereditary radical and let $R$ be a subring of a ring $S$. Then $\alpha(S) \cap R \subseteq \alpha(R)$.

PRoof. $\alpha(S) \cap R \subseteq \alpha(S) \in \alpha$, so $\alpha(S) \cap R \in \alpha$ since $\alpha$ is a strongly hereditary radical. But $\alpha(S) \cap R$ is an ideal of $R$. Thus $\alpha(S) \cap R \subseteq \alpha(R)$.

Our next observation, contains a helpful criterion which can be used to identify special radicals which are FCE-friendly.

PROPOSITION 5. Let $\mu$ be a special class of rings such that if $S$ is a finite centralizing extension of $R$, then for every $\mu$-ideal $Q$ of $R$ there exists a $\mu$-ideal I of $S$ such that $Q \supseteq I \cap R$. Then the special radical $\alpha=\mathcal{U}(\mu)$ is FCE-friendly.

PROOF. Since $\alpha$ is a special radical, $\alpha(R)$ is the intersection of all ideals $Q$ of $R$ such that $R / Q \in \mu$. Then, by the assumption, for each such $Q$, there exists a $\mu$-ideal $I$ of $S$ such that $Q \supseteq I \cap R$. Now, each such $I$ contains $\alpha(S)$ and so $\alpha(R)$ contains $\alpha(S) \cap R$ which shows that $\alpha$ is FCE-friendly.

To show some applications of Proposition 5, we need the following result which follows from [9, Theorem 10.2.4 and Theorem 10.2.9 and its proof]. 
LEMMA 6. Let $S$ be a finite centralizing extension of $R$. If $I \in \operatorname{Spec}(S)$, then $I \cap R$ $\in \operatorname{Spec}(R)$ and if $Q \in \operatorname{Spec}(R)$, then there exists $I \in \operatorname{Spec}(S)$ such that $Q=I \cap R$.

COROLlaRY 7. Let $\mu$ denote any of the following special classes: the class of all subdirectly irreducible rings with idempotent hearts; the class of all simple rings with identity or the class $*_{k}$. Then the radical $\mathcal{U}(\mu)$ is FCE-friendly.

PROOF. Let $S$ be a finite centralizing extension of $R$.

First we show that the antisimple radical $\beta_{\varphi}$, that is, the upper radical generated by the class of all subdirectly irreducible rings with idempotent hearts is FCE-friendly. Let $Q \triangleleft R$ be such that $R / Q$ is a subdirectly irreducible ring with an idempotent heart $H / Q$, where $H$ is an ideal of $R$ strictly containing $Q$. Then clearly $Q \in \operatorname{Spec}(R)$ and, it follows from Lemma 6, that there exists a prime ideal $I$ of $S$ such that $Q=I \cap R$. Then, by Zorn's lemma, there exists an ideal $P$ of $S$ that is maximal with respect to having $Q=P \cap R$. It is easy to check that this $P$ is a prime ideal of $S$. Moreover, it was proved in [11, proof of Proposition 3.1] that $S / P$ is a subdirectly irreducible ring with heart containing $(H+P) / P \simeq H / Q$ which is nonzero. Thus $S / P$ is a subdirectly irreducible ring with an idempotent heart which shows that the class of all subdirectly irreducible rings with idempotent hearts satisfies the condition of Proposition 5. Consequently $\beta_{\varphi}$ is FCE-friendly.

To show that the Brown-McCoy radical $\mathcal{G}=\mathcal{U}$ (all simple rings with identity) is FCE-friendly, it suffices to show that the class of all simple rings with identity satisfies the condition of Proposition 5. Let $Q \triangleleft R$ be such that $R / Q$ is a simple ring with identity. Then $Q \in \operatorname{Spec}(R)$ and, arguing as in the first part of the proof, we can find an ideal $P$ of $S$ that is maximal with respect to having $Q=P \cap R$ and $P \in \operatorname{Spec}(S)$. Then $Q \supseteq P \cap R$. We will show that $S / P$ is a simple ring with identity. Let $J / P$ be a nonzero ideal of $S / P$, where $J$ is an ideal of $S$ strictly containing $P$. Then, by the maximality of $P, J \cap R$ strictly contains $Q$, or equivalently, $(J \cap R) / Q$ is a nonzero ideal of the simple ring $R / Q$. Consequently $(J \cap R) / Q=R / Q$ which implies $R \subseteq J$ and, since $R$ and $S$ have the same identity and $J$ is an ideal of $S$, it follows that $S=J$. Thus $R / P$ is a simple ring. with identity which ends the proof.

We will now show that the class $*_{k}$ satisfies the condition of Proposition 5 . Let $Q \triangleleft R$ be such that $R / Q \in *_{k}$. If $Q=R$, then $I=S$ satisfies the conditions of Proposition 5. So assume that $Q \varsubsetneqq R$. This, in view of Theorem 1 , means that $R / Q$ is a prime ring with $0 \neq p s(R / Q)=H / Q$, where $H$ is an ideal of $R$ containing $Q$. Since such $Q$ is a prime ideal of $R$, arguing as in the first part of the proof again, we can find an ideal $P$ of $S$ that is maximal with respect to having $Q=P \cap R$ and $P \in \operatorname{Spec}(S)$. We will show that $p s(S / P) \neq 0$. Let $J / P$ be a nonzero prime ideal of $S / P$. Then $J$ is a prime ideal of $S$ strictly containing $P$ and so, by the maximality of $P, J \cap R$ strictly contains $Q$. Then $J \cap R$ is a prime ideal of $R$ and it follows that $(J \cap R) / Q$ is a nonzero prime ideal of $R / Q$. This implies that $H / Q \subseteq(J \cap R) / Q$, so $J$ contains $H$ and hence $J / P$ contains $(H+P) / P$ which is nonzero. Thus $\operatorname{ps}(S / P) \supseteq(H+P) / P \neq 0$. But, since $S / P$ is a prime ring, this, in view of Theorem 1 , shows that $S / P \in *_{k}$. Thus the class $*_{k}$ satisfies the condition 
of Proposition 5 which implies that the radical $\mathcal{U}\left(*_{k}\right)$ is FCE-friendly and ends the proof.

Our next result shows a connection between the FCE-friendliness and the Amitsur property of radicals and gives a useful criterion for identifying supernilpotent radicals with the Amitsur property. In what follows, the usual extension of a ring $A$ obtained by adjoining unity is denoted by $A^{1}$.

THEOREM 8. Let $\alpha$ be a supernilpotent radical. If $\alpha$ is an FCE-friendly and $\mathbb{Z} \in$ $\mathcal{S}(\alpha)$, then $\alpha$ has the Amitsur property.

ProOF. We adapt the proof of [11, Theorem 3.2]. First observe that, for every ring $T \neq T^{1}, T[x]$ is an ideal of $T^{1}[x]$ and so $\alpha(T[x]) \subseteq \alpha\left(T^{1}[x]\right)$. On the other hand, $T^{1}[x] / T[x] \simeq \mathbb{Z}[x]$. Since $\mathbb{Z}$ is a prime ring with identity and the centre of $\mathbb{Z}$ is infinite, it follows from [2, Lemma 5, p. 243] that $\mathbb{Z}[x]$ is a subdirect sum of copies of $\mathbb{Z} \in \mathcal{S}(\alpha)$. Hence $\mathbb{Z}[x] \in \mathcal{S}(\alpha)$. Thus $\alpha(T[x])=\alpha\left(T^{1}[x]\right)$.

Now, since $\alpha$ is a supernilpotent radical, it follows from [1] that to complete the proof, it suffices to show that if $A$ is a ring of prime characteristic $p$, then $\alpha(A[x]) \cap A\left[x^{p}-x\right] \subseteq \alpha\left(A\left[x^{p}-x\right]\right)$. Now, if $A$ has the identity 1 , the result follows because $\alpha$ is FCE friendly and $S=A[x]$ is a finite centralizing extension of $R=A\left[x^{p}-x\right]$ generated by $b_{1}=1, b_{2}=x, \ldots, b_{p}=x^{p-1}$. This is because

$$
\begin{gathered}
x^{p}=\left(x^{p}-x\right)+x \in 1 R+x R+\cdots+x^{p-1} R, \\
x^{p+1}=x\left(x^{p}-x\right)+x^{2} \in 1 R+x R+\cdots+x^{p-1} R
\end{gathered}
$$

and, it follows by the mathematical induction that $x^{p+n} \in 1 R+x R+\cdots+x^{p-1} R$ for any natural number $n$. If $A$ has no identity, then

$$
\alpha(A[x]) \cap A\left[x^{p}-x\right] \subseteq \alpha\left(A^{1}[x]\right) \cap A^{1}\left[x^{p}-x\right] \subseteq \alpha\left(A^{1}\left[x^{p}-x\right]\right)=\alpha\left(A\left[x^{p}-x\right]\right)
$$

which ends the proof.

In a private communication, E. R. Puczylowski gave the following example which shows that the Amitsur property of a radical $\alpha$ does not imply that $\alpha$ is FCE-friendly.

EXAmple 9. Consider the special radical $\alpha=\mathcal{U}(\mathbb{R})$. According to [7, Theorem 4.9.22], in order to show that $\alpha$ has the Amitsur property, it suffices to show that $\alpha(A[x]) \cap A=0$ implies $\alpha(A[x])=0$ for all rings $A$. So let $A$ be a ring such that $\alpha(A[x]) \cap A=0$ and suppose that $\alpha(A[x]) \neq 0$. Then

$$
A \simeq A /(A \cap \alpha(A[x])) \simeq(A+\alpha(A[x])) / \alpha(A[x]) \subseteq A[x] / \alpha(A[x]) .
$$

But, as $A[x] / \alpha(A[x]) \in \mathcal{S}(\alpha)$, it follows that $A[x] / \alpha(A[x])$ is a subdirect sum of copies of $\mathbb{R}$ and hence $A[x] / \alpha(A[x])$ is torsion free. Then $A$ is also torsion free and then it follows from [1] that $\alpha(A[x]) \neq 0$ implies $\alpha(A[x]) \cap A \neq 0$ which gives a contradiction. Thus $\alpha$ has the Amitsur property. However, $\alpha$ is not FCE-friendly because $\alpha(\mathbb{C}) \cap \mathbb{R}=\mathbb{C} \cap \mathbb{R}=\mathbb{R} \nsubseteq\{0\}=\alpha(\mathbb{R})$. 
COROLlary 10. If $\alpha$ is any of the following special radicals: $\beta, \mathcal{J}$, the Behrens radical $\gamma, \beta_{\varphi}, \mathcal{G}$ or $\mathcal{U}\left(*_{k}\right)$, then $\alpha$ has the Amitsur property.

PROOF. Let $\alpha$ be any of the radicals listed in Corollary 10. Since any special radical is supernilpotent, each such $\alpha$ is supernilpotent and Corollary 7 and our previous remarks imply that each such $\alpha$ is FCE-friendly. Moreover, since $\mathbb{Z}$ is the subdirect sum of the finite fields $\mathbb{Z}_{p_{i}}$, where $p_{i}$ ranges through the prime numbers and each $\mathbb{Z}_{p_{i}} \in \mathcal{S}(\alpha)$, it follows that $\mathbb{Z} \in \mathcal{S}(\alpha)$. Thus it follows from Theorem 8 that each such $\alpha$ has the Amitsur property.

Our remaining results show that $\mathcal{U}\left(*_{k}\right)$ behaves similarly to $\beta$ when one takes polynomials.

THEOREM 11. The radical $\mathcal{U}\left(*_{k}\right)$ is polynomially extensible, that is, if $A \in \mathcal{U}\left(*_{k}\right)$, then $A[x] \in \mathcal{U}\left(*_{k}\right)$ for any ring $A$.

Proof. Suppose $A[x] \notin \mathcal{U}\left(*_{k}\right)$. It suffices to show that $A \notin \mathcal{U}\left(*_{k}\right)$. Since $A[x] \notin$ $\mathcal{U}\left(*_{k}\right)$, then $0 \neq A[x] / P \in *_{k}$ for some $P \triangleleft A[x]$. Then it follows from Theorem 1 that $A[x] / P \in \pi$ and $p s(A[x] / P) \neq 0$. But this together with [3, Lemma 4.2] implies that $\operatorname{ps}(A / P \cap A) \neq 0$. But then, since $A /(P \cap A) \in \pi$, it follows from Theorem 1 that $0 \neq A /(P \cap A) \in *_{k}$. This shows that $A \notin \mathcal{U}\left(*_{k}\right)$ and ends the proof.

Corollary 12. For any ring $A, \mathcal{U}\left(*_{k}\right)(A[x])=\mathcal{U}\left(*_{k}\right)(A)[x]$.

PROOF. Theorem 11 shows that $\mathcal{U}\left(*_{k}\right)$ is polynomially extensible. Since, being a special radical, $\mathcal{U}\left(*_{k}\right)$ is also hereditary, it follows from [7, Proposition 4.9.21] that $\mathcal{U}\left(*_{k}\right)(A)=\mathcal{U}\left(*_{k}\right)(A[x]) \cap A$. This and the fact that $\mathcal{U}\left(*_{k}\right)$ has the Amitsur property imply that $\mathcal{U}\left(*_{k}\right)(A[x])=\left(\mathcal{U}\left(*_{k}\right)(A[x]) \cap A\right)[x]=\mathcal{U}\left(*_{k}\right)(A)[x]$ which ends the proof.

Corollary 13. For any ring $A$, if $A \in *_{k}$, then $A[x] \in \mathcal{S}\left(\mathcal{U}\left(*_{k}\right)\right)$.

Proof. Let $A \in *_{k}$. Then $\mathcal{U}\left(*_{k}\right)(A)=0$. Then Corollary 12 implies that $\mathcal{U}\left(*_{k}\right)(A[x])=0$ which means that $A[x] \in \mathcal{S}\left(\mathcal{U}\left(*_{k}\right)\right)$.

\section{Acknowledgement}

The author expresses her gratitude to the referee for valuable comments which improved the original manuscript considerably.

\section{References}

[1] S. A. Amitsur, 'Radicals of polynomial rings', Canad. J. Math. 8 (1956), 355-361.

[2] V. A. Andrunakievich and Yu. M. Ryabukhin, Radicals of Algebra and Structure Theory (Nauka, Moscow, 1979), in Russian.

[3] M. Ferrero and R. Wisbauer, 'Unitary strongly prime rings and related radicals', J. Pure Appl. Algebra 181 (2003), 209-226.

[4] H. France-Jackson, 'On atoms of the lattice of supernilpotent radicals', Quaest. Math. 10 (1987), 251-256. 
[5] _ 'Rings related to special atoms', Quaest. Math. 24 (2001), 105-109.

[6] B. J. Gardner, 'Some recent results and open problems concerning special radicals', in: Radical Theory, Proceedings of the 1988 Sendai Conference, Sendai, 24-30 July 1988 (ed. S. Kyono) (Uchida Rokakuho, Tokyo, 1989), pp. 25-56.

[7] B. J. Gardner and R. Wiegandt, Radical Theory of Rings (Marcel Dekker, New York, 2004).

[8] H. Korolczuk, 'A note on the lattice of special radicals', Bull. Pol. Acad. Sci. Math. 29 (1981), 103-104.

[9] J. C. McConnell and J. C. Robson, Noncommutative Noetherian Rings (John Wiley \& Sons, Chichester, 1987).

[10] N. H. McCoy, The Theory of Rings (MacMillan, New York, 1964).

[11] P.-H. Lee and E. R. Puczylowski, 'On the Behrens radical of matrix rings and polynomial rings', J. Pure Appl. Algebra 212 (2008), 2163-2169.

HALINA FRANCE-JACKSON, Department of Mathematics and Applied Mathematics, Summerstrand Campus (South), Nelson Mandela

Metropolitan University, PO Box 77000, Port Elizabeth 6031, South Africa

e-mail: cbf@easterncape.co.uk 